\title{
Intorno ad alcune particolarità del raggio verde ${ }^{1}$ ).
}

\author{
Nota DI G. GUGLIELMO.
}

Il così detto raggio verde (ossia $\mathrm{i}$ primi raggi del sole che incomincia a spuntare sull' orizzonte, e gli ultimi del sole che tramontando scompare, i quali di solito sono verdi o azzurri, spesso molto brillanti) $\dot{e}$ stato attribuito a tre cause diverse: la rifrazione e dispersione atmosferica, l'assorbimento atmosferico, una illusione ottica per effetto di contrasto.

La prima di queste cause, la cui azione non può esser messa in dubbio, è certamente la più importante, le altre due possono essere concomitanti e modificare la colorazione e forse la durata del raggio.

Il sole presso all'orizzonte, osservato con un cannocchiale di mediocre ingrandimento, e con una sufficiente diminuzione dello splendore, presenta sempre ben visibili un orlo rosso alla parte inferiore, e un orlo verde o azzurro alla parte superiore, orli che senza dubbio sono prodotti dalla rifrazione atmosferica che devia più i raggi verdi o azzurri che non $i$ rossi. I raggi indaco, violetto, e quelli ultravioletti più deviati dell' azzurro, sono completamente assorbiti quando il sole è presso l'orizzonte. L'orlo verde o azzurro, naturalmente, spunta prima, e tramonta dopo della parte bianca abbagliante del disco solare, producendo cosi il raggio verde.

F. Exner (Pernter. Meteorologische Optik, pag. 799), per spiegare la durata reale del raggio verde che di solito $\dot{\theta}$ maggiore di quella teorica, suppone che questo possa esser dovuto all'assorbimento dei raggi rossi prodotto dal vapore acqueo degli strati inferiori dell' atmosfera, dimodochè esso incomincierebbe a prodursi quando la parte bianca del disco

1) Dai Rendiconti della R. Accademia dei Lincei, Vol. XXV, serie 5. ${ }^{*}, .^{\circ}$ Sem. 1916, pag. 296. 
solare non è ancora interamente tramontata. La colorazione cosi prodotta non sarebbe visibile quando lo è una gran parte del disco solare, sia perchò questa è troppo abbagliante, sia perchè i raggi emessi dalla sua parte superiore attraversano meno obliquamente gli strati assorbenti.

Più probabile mi pare che questo assorbimento, quando si produce, sia dovuto a particelle minutissime di acqua o ghiaccio o pulviscolo, selezionate per effetto della gravità e della resistenza dell'aria in modo che in ogni strato orizzontale abbiano uguali dimensioni.

Ho osservato varie volte un effetto che pare dovuto a questa causa, (nell'ottobre del 1915̃), dal Bric della Croce, presso Torino, alto 700 metri circa sul livello del mare, mentre il sole tramontava dietro le Alpi alte circa 3000 metri; trenta secondi circa prima della totale scomparsa del sole, il segmento ancora visibile prese una tinta rosso-violacea uniforme e visibile anche nell' illuminazione circostante, ed essa andò diventando sempre più carica mentre il sole s'abbassava, finchè questo scomparve producendo un raggio verde che invece era di colore decisamente azzurro, della durata di un secondo o meno. (Questa durata è diminuita per effetto dell'attitudine dal punto d'osservazione e dell'altezza angolare della linea dei monti).

Da queste osservazioni (non ne conosco altre) ed anche dalla causa presunta risulta che questo effetto è essenzialmente diverso dal raggio verde dovuto alla rifrazione atmosferica.

Recentemente A. W. Porter (Nature, vol. 94. ${ }^{\circ}$, pag. 672; vol. 95.', pag. 194) attribui il raggio verde ad un effetto fisiologico di contrasto ed ha eseguito esperienze con un sole artificiale rosso le quali confermerebbero questa spiegazione. Si غे obbiettato (Nature, vol. 95, pag. 8) che, appunto quando il sole è rosso, il raggio verde è debolissimo o manca del tutto e che questo si osserva anche al sorger del sole quando non vi può essere effetto di contrasto; inoltre effetti di questo genere dovrebbero essere confermati da molti osservatori, per evitare le illusioni personali.

Da molti anni ho avuto frequenti occasioni di osservare il raggio verde, più spesso al tramonto, dalle finestre del Gabinetto fisico dell'Università di Cagliari; spesso anche al sorger 
del sole, dalle finestre dell'abitazione notturna, in condizioni piuttosto favorevoli perchè $i$ punti d'osservazione erano alti circa 70 e 40 metri, rispettivamente, sulla pianura interposta, ed il sole sorgeva o tramontava dietro due linee di monti, lontane parecchie decine di chilometri, alte angolarmente da mezzo grado a due gradi e mezzo, dimodochè (sebbene fosse diminuita la rifrazione) erano molto diminuite l'opacità e la poca omogeneità che la vicinanza del suolo e della città produce negli strati inferiori dell'aria. L'omogeneità dell'atmosfera era dimostrata dalla grande e costante regolarità del contorno solare').

In dicembre e in gennaio ho anche osservato il raggio verde quando il sole sorgeva sul mare. Il disco solare, in queste condizioni, appariva molto schiacciato: il diametro verticale era circa $4 / 5$ di quello orizzontale, ed il contorno della metà inferiore era assai meno curvo di quello della metà superiore; il tutto parvemi in misura maggiore di quanto corrisponderebbe alla diminuzione regolare della rifrazione al crescere dell'altezza.

Ho fatto le osservazioni del raggio verde, talora con un binoculo a prismi d'ingrandimento 9 (col quale in buone con-

1) Una sola volta, il 22 settembre 1912, osservai un effetto di miraggio. Dopo una giornata calma e calda, si era levato nel pomeriggio un freseo vento di maestrale che, suppongo. lasciava immobili ad ovest gli strati d'aria vicini al suolo e compresi fra due catene di montagne formanti come una conca. Quando l'orlo inferiore del disco solare si nascondeva già dietro i monti alti circa mezzo gradn, osservai, al disopra e molto viciıo all'orlo super ore. un segmento circolare, brillante come il sole stesso, alto circa $\%$ del diametro di questo, limitato superiormente dalla corda orizzontale, ed inferiormente dall'arco, che aveva un orlo verde, affatto uguale a quello dell' adiacente contorno solare.

A misura che il sole s'abbassava, questo segmento diminuiva d'ampiezza, perchè, mentre la corda rimaneva alla stessa altezza, l'arco s'innalzava sino a che spari producendo un raggio verde della solita durata. Questo segmento era senza dubbio l'immagine della parte superiore del disco solare, prodotta per riflessione (intensa, perchè totale o molto obliqua) o per rifrazione sulla superficie di separazione fra l'aria calda della valle e l'aria fresca degli strati superiori, superficie che era più alta del luogo dell'osservazione. 
dizioni, molto rare, erano appena visibili l'orlo rosso e l'orlo verde), talora con un piccolo eannocchiale d'ingrandimento 25 , spesso anche con un cannocchiale di Steinheil con obbiettivo di $108 \mathrm{~mm}$. di diametro e $163 \mathrm{~cm}$. di distanza focale con ingrandimenti da 80 a 400 , che rendevano visibili le particolarità dei suddetti orli colnrati.

Siccome i soliti oculari di Huygens-Mittenzwey di questo cannocchiale erano imperfettamente acromatici presso gli orli del campo (dimodochè lo spessure degli orli colorati del disco solare appariva aumentato o diminuito a seconda della loro posizione nel campo stesso), quando volevo misurare questo spessore usai due oculari acromatici, simmetrici, con foco esterno, che producevano gl'ingrandimenti di 80 e 180 rispettivamente ed erano entrambi provvisti di micrometro oculare.

Nelle osservazioni dell'or $]_{o}$ verde o azzurro è necessario d'indebolire e regolare lo splendore abbagliante del disco solare, non soltanto per non affaticare l'occhio, ma anche perchè variano così il colore ed altre apparenze dell'orlo stesso; e son ricorso perciò ai soliti mezzi.

Talora essendo il sole piuttosto alto sull'orizzonte, ho proiettato la sua immagine ingrandita, prodotta dal cannocchiale, su di uno schermo bianco; più spesso, osservando direttamente l'immagine, ho limitato la parte libera dell' obbiettivo con dischi di cartone con fori di vario diametro, da 4 ad 1 centimetro, ed anche meno se l'ingrandimento era piccolo e l'immagine più brillante. Coi fori di minor diametro era difficile di trovare la posizione più conveniente dell'oculare, che poteva esser spostato senza che la nitidezza delle immagini variasse in modo notevole '); e perciò questa posi-

4) Questa profondità di foco dei cannocchiali con obbiettivo di piccolo diametro e con lunga distanza focale (principale o solo coniuga a ), notevole specialmente con deboli oculari, puó riuscire utile in molti apparechi di fisica, quando oceorra osservare simultaneamente oggetti situati a distanze diverse (p. es. due termometri un po' distanti, oppure un indice sopra una scala un po' distante da esso, un filo a piombo e l'asta da rettificare ecc.). 
zione era determinata coll'obbiettivo interamente scoperto, osservando oggetti lontani.

Mi sono anche servito di un oculare polarizzatore sempliticato, ciò̀ munito di due soli specchi di vetro nero, uno anteriore che riceveva i raggi solari sotto l'angolo di polarizzazione totale, l'altro che riceveva i raggi riflessi pure sotto lo stesso angolo e poteva rotare. attorno al raggio centrale; l'oculare, completo e più comódo, di Merz, con la quadruplice riflessione a $45 .^{\circ}$, indeboliva troppo l'immagine del sole presso all'orizzonte.

Se il sole è ancora un po' alto sull'orizzonte (più di $\overline{\mathbf{b}}^{\circ}$ ) e molto brillante, e se l'immagine viene proiettata sullo schermo, l'orlo superiore appare verde con un po' d'azzurro all'esterno; se, invece, nelle stesse condizioni si osserva direttamente il sole senza diminuirne troppo lo splendore, l'orlo appare azzurro, ed il verde è invisibile. E chiaro che nel primo caso l'orlo azzurro è poco visibile perchè troppo debole; nel secondo caso $\dot{e}$ invisibile l'orlo verde perchè troppo debole rispetto all'adiacente ed abbagliante parte bianca del disco solare.

A misura che il sole s'abbassa sull'orizzonte, la parte azzurra dell'orlo diviene meno visibile anche coll'osservazione diretta; ed in estate finisce con lo scomparire del tutto, mentre diviene sempre più visibile quella verde.

La parte azzurra dell'orlo è sfumata verso l'esterno, senza contorni decisi. La parte verde ha contorni decisi: si presenta come uno strato di liquido verde, galleggiante sulla parte bianca sottostante, ed in continuo movimento. Si formano onde che si muovono nel senso del vento simili a quelle che si osservano in una spiaggia marina; si osservano anche nuclei rotondi a modo di goccie ed inoltre si producono intagli che penetrano un poco anche nella parte bianca e che si muovono propagandosi sempre verso l'alto e diventando più profondi, finchè, giunti alla sommità, un segmento dell' orlo verde si stacca $\theta$ s'innalza dileguandosi.

Questi movimenti, che aumentano a misura che il sole s'avvicina all'orizzonte, fanno si che lo spessore dell' orlo verde sia continuamente variabile, e che la colorazione ora si diluisca estendendosi, ora si concentri restringendosi. 
Nelle condizioni in eui osservavo, questo spessore, nell'immagine prodotta dall'obbiettivo di $163 \mathrm{~cm}$. di distanza focale, era di circa $0,1 \mathrm{~mm}$. ossia 12"; talora, anche due e tre volte di più quando l'orlo suddetto era vicinissimo alla linea dei monti e la sua agitazione era magginre.

Circostanze finora non bene spiegate del raggio verde sono: Ia colorazione varia dal verde all'azzurro; la varia intensità, talora notevole, talora pressochè nulla; e la durata, di solito maggiore di quella teorica, che viene dedotta dagli indici di rifrazione dei varî colori e maggiore anche di quella che corrisponde allo spessore osservato dell' orlo verde.

Il fatto che l'orlo azzurro è ben visibile quando il sole è ancora un po' alto sull'orizzonte, e va indebolendosi e spesso scompare quando il sole è molto basso, prova che tale indebolimento e tale scomparsa (e quindi la colorazione verde dell'orlo e del raggio estremo) sono dovuti all'assorbimento atmosferico, prodotto sui raggi solari dagli strati inferiori dell'atmosfera, dei quali lo spessore attraversato dai raggi cresco quando il sole s'abbassa verso l'orizzonte.

Solitamente (ma non sempre) d'estate, allorchè la quantità di vapore contenuta nell' aria era maggiore, l'orlo superiore del sole presso all'orizzonte ed il raggio ultimo erano decisamente verdi, mentre nelle altre stagioni erano più $o$ meno azzurri, tuttavia non credo che l'assorbimento sia prodotto per assorbimento diretto dal vapor acqueo perchè la quantità di questo si può dedurre con molta approssimazione dalla temperatura $\theta$ dal grado di umiditá; e non $\dot{e}$ in relazione costante colle colorazioni suddette.

Notisi che d'estate è maggiore anche la quantità di pulviscolo dovuta all'aridità del suolo.

Siccome i raggi del solo presso l'orizzonte subiscono sempre la rifrazione atmosferica, le cui variazioni in uno stesso luogo sono relativamente molto piccole, sarebbe da credere che il raggio verde dovrebbe prodursi sempre con la stessa intensità relativamente allo splendore del sole, mentre avviene talora che in condizioni favorevoli, cioè con aria limpida e sole bianco. e brillante, il raggio verde sia sbiadito, appena visibile, mentre avviene anche che, in condizioni che si direbbero sfavorevoli, 
cosi l'orlo verde come il raggio verde, specialmente se osservati con un cannocchiale, abbiano una bellissima colorazione.

$\dot{\mathrm{E}}$ chiaro che varie cause possono influire sull'intensiti del raggio verde: il soverchio splendore del sole, affaticando l'occhio, puó far apparire meno intenso il raggio verde; lo splendore dell' atmosfera nel punto ove esso si prodnce può diluirlo e renderlo meno evidente; le particelle di pulviscolo possono avere tali dimensioni da assorbire appunto quei raggi di cui si compone; finalmente, a causa del movimento dell'orlo verde, può avvenire che esso, nell'istante in cui si produce il raggio verde, abbia uno spessore minimo o colorazione diluita che ne diminuiscono l'jntensità. Non pare che la latitudine abbia influenza sul raggio verde, poichè esso è stato osservato cosi nel mare del Nord come nel mediterraneo meridionale. Invece, siccome è stato osservato sul mare o dal mare o in sua vicinanza, si potrebbe credere che questo vi influisca, forse come suppone F. Exner (loc. cit.), per l'abbondanza di vapor acqueo.

Sebbene le mie osservazioni siano state fatte quasi tutte in vicinanza del mare, pure ho avuto occasione di osservare il raggio verde (di colore decisamente azzurro, della durata di 2 secondi, da Firenze mentre il sole tramontava dietro una collina lontana circa $100 \mathrm{~km}$. dal mare; e come ho già detto, l'ho osservato a Torino, mentre il sole tramontava dietro i monti lontani dal mare migliaia di chilometri nella direzione del raggio e dove l'atmosfera doveva contenere assai poco vapore.

W. A. Julius (Archives des sciences nèerlandaises, serie II, tome IV) suppone $\operatorname{ch} \theta$ la lunga durata del raggio verde, spesso molto maggiore di quella teorica, sia dovuta ad una rifrazione anomala dei raggi verdi negli strati inferiori dell'atmosfera: raggi che, in proporzione col grado di ionizzazione atmosferica, subirebbero una deviazione molto maggiore di quella normale. Sebbene io abbia cercato in molti modi di constatare l'influenza di questa causa sul raggio e sull'orlo verde, non vi sono riuscito, forse per mancanza di opportuni strumenti, ma anche - credo - perchè tale influenza, se esiste, non è essenziale.

Con un prisma obbiettivo con angolo dispersivo di $10^{\circ}$, collocato dinanzi all'obbiettivo del cannocchiale, la dispersione 
ottenuta era troppo grande, rispetto a quella atmosferica; e così pure avveniva con un oculare spettroscopico.

Con un disco di cartoncino perforato Bristol, collocato dinanzi all' obbiettivo come consiglia lo Henry (Comptes rendus, vol. 112 , pag. 377) si otteneva per diffrazione un effetto troppo complesso; e cosi pure, sebbene in grado molto minore, con un reticolo di diffrazione formato con fili di acciaio di $1 \mathrm{~mm}$. di diametro, fra $\mathrm{i}$ quali, alle estremità, erano interposti corti tratti dello stesso filo producenti intervalli di $1 \mathrm{~mm}$.

Ho costruito in seguito un prisma ad acqua con angolo dispersivo piccolissimo, tale che la distanza angolare delle righe $C$ ed $F$ fosse uguale a quella prodotta dalla dispersione atmosferica. Due lamine di vetro di $4 \mathrm{~cm}$. di lato, a faccie otticamente piane e parallele, erano sovrapposte in modo che fossero a contatto secondo uno dei lati e fossero separate lungo il lato opposto da due tratti di filo di rame, spesso 0,4 oppure $0,5 \mathrm{~mm}$; esse erano masticiate agli orli, lasciando un foro per il quale veniva introdotta fra mezzo l'acqua distillata.

Adattato questo prisma entro un foro quadrato in un disco di cartone spesso, a collocatolo dinanzi all'obbiettivo, se lo spigolo era verticale, la dispersione orizzontale del prisma e quella verticale dell'atmosfera si componevano, producendo gli orli opposti rosso e verde nel contorno del sole a $45^{\circ}$, mentre una dispersione anomala notevole dei raggi verdi avrebbe dovuto spostare seusibilmente l'orlo verde verso l'alto

Disposto orizzontalmente ed in basso lo spigolo del prisma, in modo che le dispersioni del prisma e dell'atmosfera fossero opposte, essendo il sole alto circa $\overline{5}^{0}$ sull' orizzonte, l'orlo superiore del sole appariva rosso quello inferiore verde o azzurro, entrambi sottili; a misura che il sole s'abbassava, comparivano corti tratti verdi nell'orlo superiore, rossi in quello inferiore, variabili, e i due orli avevano all'incirca la stessa apparenza quando il sole era presso l'orizzonte, ciò che prova l'uguaglianza delle due dispersioni.

Notevole era anche, che mentre osservando col cannocchiale, senza il prisma, il contorno della parte bianca del sole in alto e in basso appariva tutto corroso, dentellato, sforac- 
chiato, col prisma appariva continuo e regolare, dimodochè mi pare che il prisma stesso giovi non poco a migliorare l'immagine degli astri presso all'orizzonte.

Credo che varie cause possano produrre la durata eccezionale del raggio verde.

Il movimento ondoso dell' orlo verde ne fa variare continuamente lo spessore, e può quindi produrre un aumento o una diminuzione del raggrio verde, ma di solito sorprende di più l'aumento. Similmente, se il sole tramonta (o sorge) nel mare, il moto della superficie di questo, sollevantesi e abbassantesi per effetto $d i$ onde che possono essere invisibili ad occhio nudo e senza un punto fisso di riferimento, può causare un aumento o una diminuzione della suddetta durata, secondo che il moto dell'onda è d'accordo o in disaccordo col moto del sole.

$\dot{\mathrm{E}}$ anche possibile che, per effetto di più masse d'aria aventi alternativamente densità maggiori e minori di quella media, il raggio che le attraversi subisca una rifrazione quindi una dispersione maggiore di quella teorica, che corrispondo ad una variazione regolare della densità coll'altezza. Cosi si spiegherebbero certe deformazioni del contorno solare, la sua ellitticità talora maggiore, la curvatura inferiore minore di quelle teoriche.

Finalmente, se il sole tramonta dietro una linea di monti, è chiaro che la durata del raggio verde sarà minima se essa linea, nel punto ove esso si produce, è perpendicolare al moto del sole; e sarà grandemente aumentata se gli è parallela. In queste ultime condizioni ho osservato durate di circa 10 sesecondi.

Il raggio verde (o rosso) si può produrre artificialmente o ripetutamente a varie altezze coprendo metà del foro del diaframma di campo (sul quale si colloca il micrometro oculare) con un mezzo dischetto opaco, e dirigendo il cannocchiale in modo che il sule vada a scomparire o comparire dietro l'orlo diametrale. Le durate del raggio verde cosi prodotte sono all'incirca uguali a quelle che si osservano nel raggio verde se l'altezza del sole è la stessa. 